\title{
Technologies in Exertion Games
}

Florian 'Floyd' Mueller'

${ }^{1}$ RMIT University, Melbourne

\section{Introduction}

Sports and technology have a long history of merging together fruitfully.

For example, technology has been very successful in offering sports people better and more nuanced feedback of their athletic performance.

Advancements in sensor technology have been providing athletes with information about their abilities, skills and achievements in ways not previously attainable without technology. In particular, technology has served as a tool for sports people to gain insights into how to improve their future athletic performances. Technology as tool has been used for training purposes by athletes, but also by coaches, trained to interpret the data in order to provide enhanced feedback for performance improvement.

Technology systems have supported sports activities for many years, however, in recent years new interactive technologies have emerged that have the potential to radically change how we understand and participate in physical activity, thereby changing the face of sports. This trend has been fuelled by the rapid rise of computer gaming advances, resulting in novel 
gaming experiences that involve physical activity in ways not available to users until recently. We call these emerging systems "Exertion Games" (Mueller et al., 2011). Exertion Games are digital games that require physical effort where the outcome of the game is predominantly determined by this physical effort, most often enabled through gross-motor abilities. This coming together of sports and computer games and associated technologies has often been described through a portmanteau of "exercise" and "gaming": exergaming (Sinclair, Hingston, \& Masek, 2007). These new technology systems borrow both from sports and computer gaming, facilitating new experiences that are often neither sports nor gaming alone, but rather a novel coming together of both.

Through analysis of existing systems and their conceptual and technical beginnings, three main approaches towards the design of these systems can be identified. Such an investigation can be useful as a guide to articulate current understandings, identify emerging opportunities, and to inspire future explorations to direct where this field should be heading. However, due to the young age of the field as well as the multidisciplinary backgrounds involved, these approaches do not mark discrete categories, but rather overlapping ones. This is acceptable as it also highlights where approaches can draw from one another. It should be noted that despite the particular articulation of the technology involved, the listing of approaches should primarily be understood as offering different perspectives towards 
the goal of offering and facilitating experiences that aim to highlight the role of the body in our interactions with technology. In consequence, the ultimate goal is to foster a heightened awareness and deepened appreciation of the active human body in society as inspired by the celebration of the active human body in sports activities.

The next section describes the three main approaches where efforts emerged in bringing sports and computer gaming together.

\section{Computer games that utilize exertion}

Computer games that utilize exertion are one category of exertion games. These systems are often game consoles that incorporate additional sensing capabilities in order to detect gross-motor movements, complementing or replacing the traditional button-presses on a gamepad controller. All three major game consoles have recently undergone such a transformation from predominantly gamepad-controlled game input console to game system that can consider gross-motor movements.

Nintendo took the lead out of the three major game consoles to date and introduced the Wii game console (Nintendo, n.d.) that uses up to four accelerometer-equipped handheld "Wiimotes" to support gross-motor movement as gaming input. Gamers and game developers alike criticized the limited sensing capability of the Wiimotes, so that Nintendo now offers 
attachments to the Wiimotes that enhance their accuracy: the "Wii Motion Plus" incorporates a "dual axis tuning fork gyroscope and a single-axis gyroscope which can determine rotational motion, allowing for more complex movements than with the original device" (Wikipedia contributors, 2011). The Wii Motion Plus promises enhanced gameplay experiences particularly when engaging in games such as tennis competitions, where players control their avatars on a tennis court by flailing their arms, similar to swinging a tennis racquet (EA Sports, 2010).

The Wii console can also be connected via Bluetooth to the Wii balancing board, which uses four pressure sensors underneath to measure the user's center of balance and weight. The Wii balance board has been used as input for game experiences, such as one where the player needs to balance the avatar on a virtual ski jump to reach maximum performance, but it has also been used to advise the correct execution of specific exercise actions such as yoga poses.

Sony's Playstation introduced a digital camera that, combined with computer vision and gesture recognition, allowed players to interact with games using motion and colour detection (eyetoykinetic.com). Next, in response to Nintendo Wii's success, Sony added the "Move" component to the Playstation, consisting of handheld controllers for each player, similar to the Wiimotes. In addition to the inertial sensors in the handheld controllers, 
a digital camera also tracks the controllers to use the position information for enhanced gameplay.

Then followed the Microsoft Xbox periphery "Kinect" (Microsoft, 2010). It consists of a digital camera and a depth sensor that allows for the motion capture of several players. The 3D depth information is achieved with an infrared laser grid projected by the device and captured by a CMOS sensor, providing game developers with an easily accessible depth map.

In sum, all three game systems allow game designers access to sensor data that can capture gross-motor movements in order to offer novel game experiences. Even though these systems utilize different technologies, they all facilitate physical effort investment used to control virtual game elements on a large screen in front of the players. In consequence, games emerged that offer novel game experiences, for example sword fights in which players assume fictional characters and engage in competitive activities they cannot experience in real life. There are also games that lean more on traditional sports activities, for example boot camp-like exercises that a virtual trainer instructs, and, if performed by the player appropriately (as determined by the sensors), also applauds, offering virtual praise as a result to physical effort. However, it should also be noted that not all of these games facilitate intense physical effort even though they are making use of the sensing technology. For example, studies showed that some of the 
most popular games for the Nintendo Wii do not require as much physical effort as the traditional sport they are taking inspiration from such as ten-pin bowling (Graves, Stratton, Ridgers, \& Cable, 2007; Lanningham-Foster et al., 2006).

Furthermore, it should be noted that these game systems have been criticized for their potential to allow players to "cheat", for example the Wii Tennis game can also be controlled by small wrist movements rather than full arm swings, consequently requiring less energy expenditure. The question of whether this is cheating or just a smart way to "work" the equipment (an approach not uncommon in traditional sports) is an area for future research. Nevertheless, Nintendo considered this opportunity a danger for their success and consequently introduced the Wiimote Plus to limit this kind of user behaviour enforcing technically that only gross-motor movements lead to success in the game.

Lastly, these types of exertion games also introduced new types of injury to the repertoire of accidents that can result from physical activity. For example, players had let go of their Wiimotes when playing Wii Bowling, effectively throwing the device at their TVs and breaking it. Excessive use of the Wiimotes has also led to the term "Wiiitis", describing injuries resulting from playing too much Wii Tennis (Bonis, 2007). 
An earlier example of an exertion game of this category is "Dance Dance Revolution" (Behrenshausen, 2007). Unlike the console systems mentioned above, Dance Dance Revolution is a dedicated arcade system that allows for only one type of game: two players have to follow dance moves displayed as arrows in the form of directions on a screen, while touch sensitive tiles measure their foot steps in order to provide visual and auditory feedback to how "well" the players dance. Dance Dance Revolution was also one of the first systems that suggested the social potential when introducing exertion to gaming: Dance Dance Revolution has been shown to facilitate social play through its performative element that is afforded by the expressiveness of the gross-motor movements involved (Behrenshausen, 2007).

It is interesting to note that the game setup with a screen in front and the specific characteristics of the sensors involved affect the way the game unfolds for the players. For example, seen from a social perspective, the technology affects how users are interacting with one another. Firstly, if players need to face a screen, they do not face each other, quite different to many traditional sports activities. Secondly, if cameras are involved, they often need to deal with an obtrusion problem, in other words the sensing cameras work best if players are standing next to each other while not touching, again this is different to most sports, in particular contact sports. In sum, the technology involved affords certain player interactions that can be seen to be more akin to computer game rather than sports experiences. 


\section{Augmented exercise systems}

Another category of exertion game systems emerged from the augmentation of exercise equipment. Exercise equipment, such as often featured in gyms, increasingly features sensing technology, most recently also enhanced with interactive technologies that derive from gaming advances, allowing for the incorporation of gaming elements to the exercise experience.

For example, the Tacx system (Tacx, 2009) is an augmented exercise bike that not only provides riders with feedback on their pace but also allows for races in virtual worlds with other riders who could be anywhere in the world as they are connected over the internet. Similar systems exist for other parallel play experiences such as rowing and stepper exercise systems (Gamersize, n.d.).

The Google Maps treadmill ("iFit," 2010) is not a game per se, but allows for a playful jogging exploration through digital representations of existing neighbourhoods: the treadmill measures a runner's performance to control a virtual jog through any city in the world via Google Maps, where the Google Street View data is displayed on a screen attached to the front of the treadmill. Runners therefore have the ability to explore a city, even one they have not been at before. For example, they can explore the Boston Marathon route from the comfort of their home. Enhanced actuating technology can 
even allow adjusting the speed of the treadmill in order to simulate the increased physical effort demands when runners are facing an incline in the form of a hill.

These augmented exercise systems usually started off as un-augmented equipment that affords certain exercise activity. Designers often aim to increase the richness of the associated experiences through the addition of interactive technology that enables gameplay. For example, by allowing users to measure and hence quantify their performance and subsequently compare it with other users, designers can facilitate competitive behaviour that is considered to be socially richer than performance activities that cannot be compared (Kretchmar, 2005). Supporting competitive behaviour can facilitate increased effort investment (Weinberg \& Gould, 2006), but also possibly even more important from a user experience standpoint, facilitate a richer experience than exercising without it (Kretchmar, 2005). Richer experiences are believed to contribute to participants' engagement, which ultimately can positively influence players' participation. In other words, it is hoped that exercises that are more fun will be engaged with longer, with more effort, and more often.

\section{Ubicomp systems}

Ubicomp stands for ubiquitous computing, and depicts a view on interactive technology that is pervading everyday life (Weiser, 1993). One part of this 
vision is based around the idea of small mobile devices that provide users with context-relevant information anytime and everywhere. Although it has been argued which parts of this Ubicomp vision have been realized and which ones have not and why not (Dourish \& Bell, 2011), the success of mobile phones, in particular smart phones, is generally considered one of the most buoyant success stories of this vision.

There are more mobile phones in the world than computers, and the rise of smart phones enables much of traditional computers' abilities to be performed by people on the go. The increase in CPU power provided by mobile phones not only allows to perform tasks that have previously been the domain of desktop computers, including the running of computer games, but they also feature additional sensing and actuating technology traditionally not included in desktop computers. For example, mobile phones know where they are through GPS, know how they are physically handled by its users through accelerometers, and can alert users even though they are not looking at the device through vibrotactile alerts. These technologies afford interaction opportunities that, in combination with the portability of the phone, have been appropriated for activities that involve exertion, such as enhancing exercise activities, but also enabled novel sports experiences.

Nike+ 
The most famous example of this category is probably the Nike+ running system developed with Apple (Apple, n.d.). The Nike+ uses a sensor in the shoe and GPS data to track the speed and location of a jogger. This is then visualized using appealing performance graphs that users can explore at the end of their runs. The joggers can also upload their data to a server, which offers the opportunity to engage in "virtual races" with other users, supporting competitions such as "who runs the most miles this week" or "who reaches an accumulated 100km first". Through the use of a heart rate sensor belt that communicates with the user's mobile phone, joggers can even track their heart rate during their exercise, learning about how their bodies respond to physical activity. Several similar systems exist, such as MapMyRun (mapmyrun.com, 2010) and RunKeeper ("RunKeeper," 2011), offering even additional features, such as the sharing of challenging running routes captured via the GPS data, promoting the explorations of the local area to further enrich the exertion experience. For example, users can leave notes online for each other on how they enjoyed each route, supporting the emergence of a social experience even though the participating joggers might have never met in person and usually run alone. Other social opportunities, again borrowing from computer gaming, are the ability to share performance data and personal assessments such as "today was a beautiful run, very happy with myself" via Twitter. Feedback from the recipients of a social network broadcast, such as an automatic post on Facebook, is also possible. For example some jogging apps allow Facebook 
friends who are online (and might not necessarily run themselves, but could be sitting at a desk) when such messages arrive in their inbox to send motivational chants back, which are then transmitted to the runner's mobile phone, delivered to the run while it is happening to enrich the running experience and motivate the jogger.

While the Nike+ system aims to enrich the existing jogging activity through ubiquitous technology, other systems such as Zombie Run ("Zombies, Run!," 2011) and Mobile Adventure Walks ("Mobile Adventure Walks," 2011) use the same technology to enable novel game experiences that is controlled by physical activity. Zombie Run is an adventure game that uses location-based data as input to a game on a mobile phone where the aim is to escape from virtual zombies that inhibit a representation of the physical world, for example a downtown area of a city. Players engage in a hide and seek game experience, where the technology enhances this activity by introducing virtual game elements. This expands the physical world dimension of the activity through the use of interactive technology. These games have also been labelled Pervasive Games, which are characterized by this expansion of the physical dimension (Montola, Stenros, \& Waern, 2009).

Other games that fall into the category of ubiquitous games are exploration games, for example games that turn an educational tour of a city into an 
interactive game where the informative content is weaved into a narrative that is informed by users' locations and interactions with the city's features (Ballagas et al., 2007).

The aforementioned examples show how sensing and actuating technology offered by modern mobile phones allow the bringing of game elements and sports activities closer together, each benefiting from the other's strengths. This relates to a larger innovation agenda that aims to utilize game elements in non-game tasks, often dubbed as "gamification". Although gamification has been criticised for being limited in adding to an understanding of computer games (Robertson, 2011), the opportunities of considering games have been embraced by industries that previously have not been working in a games context (Deterding, Dixon, Nacke, O'Hara, \& Sicart, 2011), essentially expanding the areas where computer games can have an impact, including in exercise and sports.

\section{Advantages of combining computer games and sports}

The aforementioned examples illustrate some of the advantages that arise when computer games, and in consequence computer gaming technologies, are combined with sports and exercise activities. The following list highlights some of the more prevalent advantages to date, however, others might emerge in the future. 
- Over a distance: One of computer games' strength is to support players in locations far apart through networking advances, allowing participants to play over a distance. This is different to traditional sports, where players mostly have to be collocated. By enabling such distributed play in sports, novel experiences could emerge that do not limit participation to users who are co-located.

- Scale: Computer games have also demonstrated that it is possible to enable play for a large number of users, for example World of Warcraft has thousands of players at any one time. Sport activities on the other hand have traditionally only supported a limited number of participants at a time. The networking advances mentioned under over a distance might also enable an increase in scale in terms of number of players.

- Asynchronicity: Traditionally, most sport activities require athletes to participate at the same time. Now interactive technologies allow comparisons over time such as with the Nike+, and most recently more advanced systems support even more complex game interactions in an asynchronous manner, for example the training of athletes across time zones (Sheridan \& Mueller, 2010).

- Balancing: In many traditional sports activities, physical strength and skill are benefitting the outcome, and if more than one player is involved, the one with more strength and higher skill is often gaining an advantage (Weinberg \& Gould, 2006). This means that if 
participants of different physical capabilities want to engage in a sports activity together, it creates an unbalanced setup that can often be detrimental to the experience. Computer games, on the other hand, have previously been successfully addressing unbalanced setups in two ways: first, differences in physical strength do not play much of a role in most computer games, so any imbalance is not a major impact on a player's experience. Secondly, differences in players' skills, such as how fast they can operate a joystick or how much experience they have in controlling a virtual character are often addressed by means of "balancing": players either select a level suitable to their skills (such as "beginner", "advanced" or "expert"), or the game software uses algorithms to balance players on the fly through dynamic difficulty adjustment (Hunicke, 2005). The result is a balanced game where players might or might not be aware of an unbalanced setup. This is done so that players feel they engaged in a "fair" experience. Balancing in sports exists only in a few selected activities, such as golf, and is always determined upfront, not allowing for changes throughout the activity, or on the fly, as in dynamic difficulty adjustment. Exertion Games can widen the use of balancing in sports by introducing levels and dynamic difficulty adjustments that consider physical effort.

- Changing the nature of the experience: Interactive technologies also introduce the opportunity to change the nature of the sports 
experience itself. For example, the use of heart rate sensors allows the changing of the nature of many sports exercises from athletic performance to effort orientation, all while not affecting the physical activity mechanic itself. Similar to balancing, the technology allows the reduction of the impact bodily abilities have and contribute to the experience. This could be desirable, for example, if participants with limited physical abilities, whether they are unfit or have a disability, would want to be made aware of their achievement regardless of their handicaps. Their performance might be low when compared to other people's performance, but making them aware that their effort investment was equal or even superior to their peers might be beneficial and motivating further effort investment.

- Fantasy: Computer games technology can also introduce a stronger fantasy element into sports. Although sports can be seen as an activity separate from "real" life, in which different rules and assumptions are in place, spanning a magic circle around the activity (Salen \& Zimmerman, 2003), computer games have demonstrated that, in particular through graphics and sound, they can facilitate a strong fantasy element. Computer games enable fantasy worlds where players are often engaged in pretend-play, for example by controlling avatars in the form of elves or dragons. They act in virtual environments that are created by game developers, but also by the players themselves, constantly adapting to their actions. Such 
opportunities as enabled by the interactive technology could be brought to the world of sports with the result of enabling novel fantasy experiences that engage players physically.

\section{Will this merging of sports and games change digital play?}

The research conducted in this area suggests that this merging of sports and computer games can and will affect how people play with digital technology. This includes positive and negative effects; the following highlights some of the more prevalent ones that have emerged so far.

- Digital game play becoming more expressive. As the body is a very expressive medium, and exertion games foster the use of the body more than most traditional computer games with their button presses, research has already identified that with the advent of sensor technologies arrived a genre of games that are more expressive (Bianchi-Berthouze, Kim, \& Patel, 2007; Juul, 2009). Players of these games have explored this expressiveness and used it to enhance their gameplay experience (Behrenshausen, 2007). By allowing and encouraging players to use their bodies in these games to express themselves, computer games can benefit from being seen as a more expressive medium. Game designers can facilitate this through their designs, for example Guitar Hero awards extra points if players move their plastic guitars like a rock star, rewarding expressive behaviour (Hero). 
- Serious games. Serious games are digital games that serve a purpose beyond mere entertainment. As such, exertion games can contribute to this agenda, mainly by offering health benefits to users afforded by the game. By providing more examples of games that serve a purpose beyond entertainment, exertion games could contribute to a heightened appreciation of the benefits of games in society. In turn, this might change players' attitudes towards games, and consequently how and why they play, affecting (hopefully positively) the role games play in people's lives.

- Games as treatment. In addition to physical health, exertion games have also been suggested to contribute to social and mental health (ERCollab: Exergaming Research Collaboration). In consequence, awareness of this might change the way players treat these games and how they engage with them. Games used in rehabilitation provide early examples of the potential power of games to form part of medical treatment (Duckworth, 2010). Sports activities have been used previously to address health issues, so it seems natural to expect similar benefits when sports and digital games come together. The coming together of sports and digital games might also change how people will experience sports in the future.

\section{Will this merging of sports and games change sports?}


Research suggests that the coming together of sports and computer games might affect how people experience sports. In particular, it has been suggested that this coming together enables to attract a new audience that has previously not been perceptible to sports activities. For example, the Nintendo Wii suggested that computer games could attract a new audience to engage in physical effort that has previously not been attracted to sports or has not been able to engage in it (Juul, 2009). Self-reports exist of users who wanted to engage in exercise, mainly for health reasons, but did not feel attracted to conventional gyms and other sports games. However, they found playing with the Nintendo Wii appealing and appropriated the associated games for their health purposes, resulting in weight loss (DeLorenzo, 2007). So far, four main reasons have emerged as to how the merging of sports and computer games could attract a new audience.

- Feedback. Digital technology can provide more accurate, nuanced and detailed feedback than previously accessible in sports. Combined with computer game elements, this feedback can be delivered in an entertaining way that many users find engaging. Furthermore, the game can adapt to this feedback, fostering an experience that aims to facilitate participants to be in the "flow zone", the optimal zone between perceived challenges and skills (Csikszentmihalyi, 1990).

- Looks like a game, not sports. Although many games for the Nintendo Wii took inspiration from existing sports, several game 
titles do not look like any existing sports game, for example, in some games players have to shake paint cans or balance dinosaurs (Nintendo). These games might be appealing to players who have a predisposition to sports, but an affinity to games. The result might be that users are "tricked into" participating in physical activity, as they might perceive their activity as gaming rather than exercise.

- Readily playable. Exertion games in the form of consoles allow exercising without the logistical burden of travelling to a gym or outdoor park. Furthermore, networking advances allow exercising with others without the need to travel first. Some might even argue that the monetary costs for participation might be lower than with traditional sports activities.

- Addressing body image issues. People with weight issues, who might be suitable users of exertion games, can experience body issues due to the way their bodies appear to others (Weinberg \& Gould, 2006). As a result, they might not feel comfortable exposing their body to physical activity in front of others, as it is the case in most gyms. Exertion games can allow exercising with others without the need to make their bodies visible to others, for example an Exertion Game in a distributed setting could refrain from conveying videoconferencing data, so that participants with body issues do not need to expose themselves in a way they are not comfortable with. Furthermore, players could assume different body conditions using 
avatars in order to "test-drive" a different body. For example, Fox and Bailenson have shown that joggers running with a digital representation of their body in the form of a 3D avatar that is slightly thinner than themselves motivates them to jog faster, "chasing" their ideal weight (Fox \& Bailenson, 2009).

This section highlighted the opportunities and consequences when sports and computer gaming come closer together. The next section explores how some of the associated prospects could come to light in concrete design systems, illustrated through a case study. The case study describes Jogging over a Distance, a project that came out of exertion games research and was conducted with the University of Melbourne, Australia, Distance Lab, UK, and London University, UK and Frank Vetere, Martin Gibbs, Stefan Agamanolis, Jennifer Sheridan and Darren Edge.

\section{JOGGING OVER A DISTANCE}

Jogging over a Distance is used as case study to illustrate some of the thinking expressed in this article. It draws on the ubiquitous computing paradigm by utilizing mobile technology for joggers, including the sensing of heart rate data. It also employs networking advances to connect users over large distances, and also the idea of balancing users of different athletic abilities (Mueller, O'Brien, \& Thorogood, 2007; Mueller, Vetere, Gibbs, 
Agamanolis, \& Sheridan, 2010; Mueller, Vetere, Gibbs, Edge et al., 2010; Mueller et al., 2012) (Fig. 1).

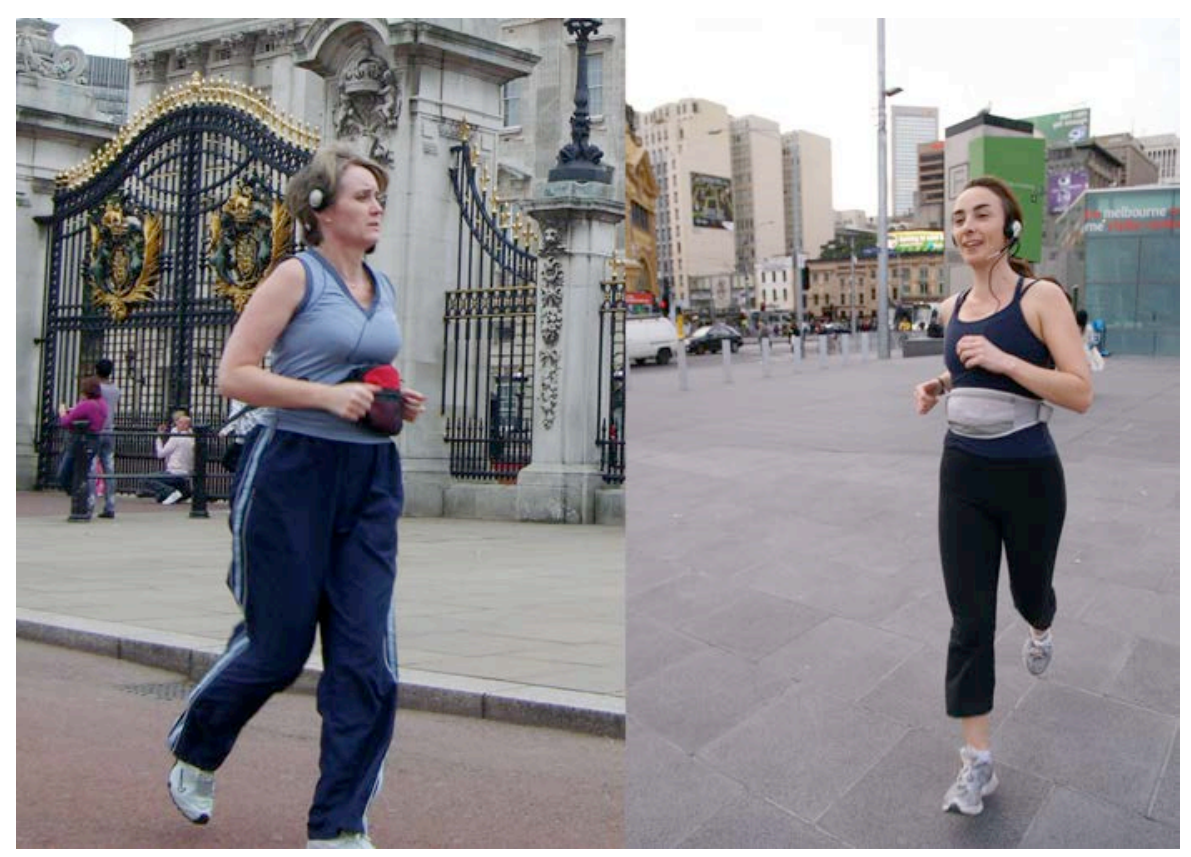

Figure 1: Jogging over a Distance between London, UK and Melbourne, Australia

Jogging over a Distance was designed to allow social joggers - these are casual joggers that enjoy the social interaction that comes with the activity to jog with their friends even though they might have moved apart. Jogging over a Distance also changes the focus of the exercise activity from performance outcome to physical effort investment through the use of heart rate data, resulting in the ability to run with friends who have very different physical abilities. For example, the system successfully supported people with very little, and some with extensive jogging experiences (such as half- 
marathons) while engaging them in a jog “together". In essence, the distance between the joggers was not something the technology needed to "fix", but rather an opportunity for the joggers to engage in an experience they could not have without the system. The next section describes this Jogging over a Distance experience.

\section{The Jogging over a Distance experience}

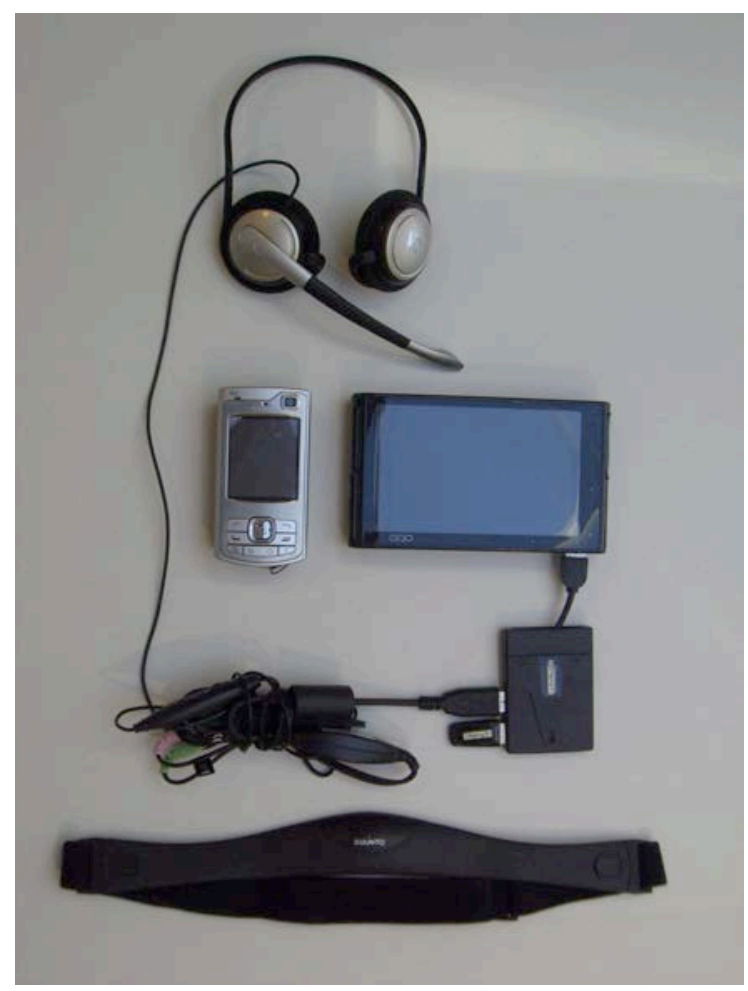

Figure 2: Jogging over a Distance's mini-computer, mobile phone, headset and heart rate belt as worn by the jogger

The Jogging over a Distance experience is as follows: Two jogging partners arrange to run at the same time (Fig. 3). Each jogger wears stereo 
headphones and a microphone as well as a heart rate monitor strapped around their chest underneath their clothing. They also wear a small pouch around their waist, which contains a mini computer and a mobile phone (Fig. 2). Although the audio could be transmitted via VoIP, the mobile phone voice channel helps keep latency to a minimum. Before the run, the system prompts users to enter their preferred target heart rate - the baseline , which allows users to specify the physical effort they plan to invest based on their fitness levels and goals.

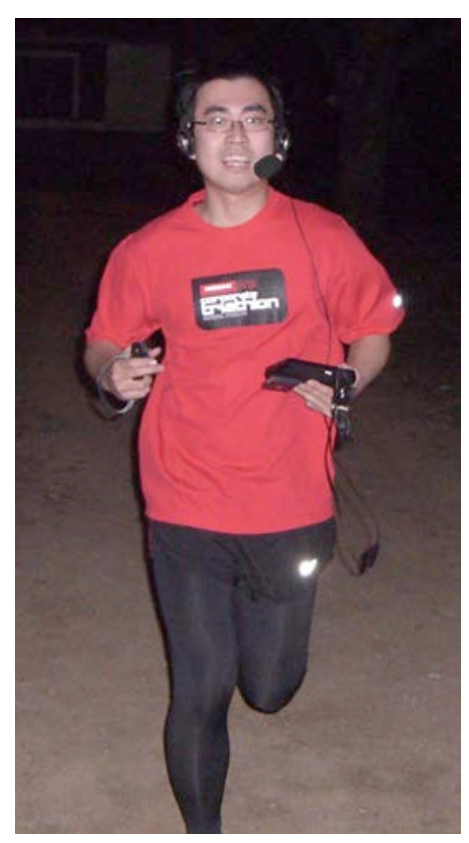

Figure 3: A jogger running at night due to the time differences

While the participants jog, their heart rate data is sent via the mobile phone's network connection to a server, analyzed and then sent to the other jogger. Each jogger can hear the audio of their jogging partner through the microphone they are wearing. The participants' heart rate data in relation to 
their baseline affects the position of the audio in a $2 \mathrm{D}$ plane that is oriented horizontally around the jogger's head. The spatialization is achieved through binaural software implemented on the mini computer that places the sound source in a 2D audio environment, moving around the jogger's head from the front, to side-by-side, and to the back depending on the difference of the relative heart rates. If the other jogger is "in front", the sound appears to come from the front, and the further "in front" the jogger is, the softer the volume of the audio. This software simulation is achieved through headrelated transfer functions and reverberation (Begault, 1994), changing the mono sound coming in through the remote microphone to create the illusion of the sound source placed in space around the local jogger, as inspired by the audio behaviour when jogging in a co-located setting. Etter (Etter, 2005) found that spatialized audio can support people running around outdoors, however, running aggravates sound localization (Marentakis \& Brewster, 2006), so to overcome this problem, the spatialization effect is amplified as described previously (Mueller, 2009).

The spatialized audio provides an indication of the relative heart rate of the jogging partner. When both joggers are at their preferred heart rate, they hear the audio coming from right beside them, as if they were running sideby-side. The same applies when both joggers divert from their preferred heart rate at the same percentage, for example, if both joggers raise their heart rate to $110 \%$, the audio stays in the center. However, if one of the 
partner's heart rate increases, the audio sounds as if it is moving ahead of the other runner, or if the heart rate decreases, the audio sounds as if it is coming from behind the jogger. For example, if the remote jogger raises her heart rate to $120 \%$, her voice coming through the headphones is softer in volume and appears to come from the front, thanks to the spatial audio. This way, the jogger is able to detect whether their partner is putting in more, the same, or less, effort, based on the relative heart rate. The system lets the jogger know when he or she needs to speed up or slow down in order to "stay" with their partner (Fig. 4).

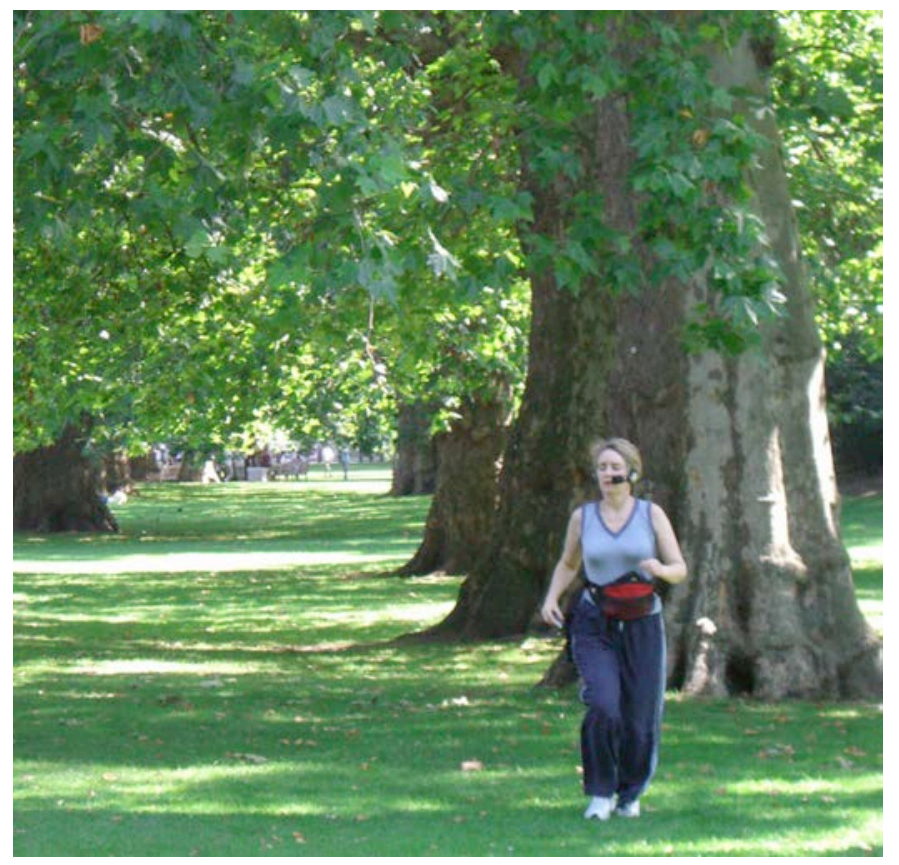

Fig. 4 Jogging alone, yet together through technology

\section{How sports and computer games come together in Jogging over a}

\section{Distance}


Jogging over a Distance benefits from the advances in ubiquitous computing in the mobile phone design space. Miniaturization of technology enables the computationally expensive spatialization software to run on small devices participants can carry. Furthermore, pervasive mobile connectivity allows the transmission of heart rate as well as voice data outdoors, such as in public parks. The ability of mobile devices to read auxiliary sensing data via Bluetooth such as heart rate is another opportunity offered by recent technology advances.

The design of the Jogging over a Distance system highlights the potential of technology to support distributed participation. Furthermore, although not demonstrated with the system, it is easily conceivable that the system could be extended to support more than two joggers at the same time, extending the notion of scale. On the other hand, how the system would support asynchronous interaction, allowing joggers to run at different times is more difficult to imagine. A combination with some of the features of the Nike+ system might offer such functionality.

Jogging over a Distance demonstrates the ability of the technology to balance players of different physical abilities (Mueller et al., 2012). One can even argue that this contributes to the fantasy element, allowing participants to imagine how it would be running with a partner who has the ability to run much faster. 
Through the heart rate, the nature of the experience also shifts from a performance-oriented activity to a more effort-focused activity. Such a shift can facilitate more intricate knowledge of how one's body responds to physical exercise, possibly fostering the development of kinaesthetic knowledge (Sheridan \& Mueller, 2010).

\section{CONCLUSION}

This chapter has offered a view on sports technology from a perspective of the coming together of sports and computer gaming, highlighting several directions of technology developments where this merging can be attributed to. This article also detailed opportunities arising from this, pointing to the unique novel experiences gaming technology can bring to sports. However, the article also points out issues such as how this coming together might affect how players experience both computer gaming and sports. Lastly, this article presented the case study of Jogging over a Distance to illustrate the conceptual points being made, substantiating the argument that the future of the coming together of sports and computer gaming is possible today and holds many opportunities for participants to engage in novel exertion game experiences. In consequence, participants have the chance to engage in experiences that can facilitate many health benefits.

\section{ACKNOWLEDGEMENTS}


The author wishes to thank the Australian Research Council, Fulbright,

Telstra, the University of Melbourne, Microsoft Research Asia,

GamesMatter, Wouter Walmink, Eberhard Graether, Chad Toprak, Josh

Platt, Sara Price, Shannon O’Brien, Alex Thorogood, Tomoko Hayashi,

Matthew Karau, Andrea Taylor and Kerin Bryant as well as all the joggers.

\section{REFERENCES}

Apple. (n.d.). Apple - Nike + iPod. Retrieved from http://www.apple.com/ipod/nike Ballagas, R. A., Kratz, S. G., Borchers, J., Yu, E., Walz, S. P., Fuhr, C. O., et al. (2007). REXplorer: a mobile, pervasive spell-casting game for tourists. ACM.

Begault, D. (1994). 3-D sound for virtual reality and multimedia: Academic Press. Behrenshausen, B. G. (2007). Toward a (Kin) Aesthetic of Video Gaming: The Case of Dance Dance Revolution. Games and Culture, 2(4), 335.

Bianchi-Berthouze, N., Kim, W., \& Patel, D. (2007). Does Body Movement Engage You More in Digital Game Play? and Why? In A. Paiva, R. Prada, \& R. Picard (Eds.), Affective Computing and Intelligent Interaction (Vol. 4738, pp. 102-113): Springer Berlin / Heidelberg. Retrieved from http://dx.doi.org/10.1007/978-3-540-74889-2_10

Bonis, J. (2007). Acute Wiiitis. New England Journal of Medicine, 356(23), 24312432.

Csikszentmihalyi, M. (1990). Flow: The psychology of optimal performance: New York: Harper and Row.

DeLorenzo, M. (2007). Wii Sports Experiment. Retrieved from http://wiinintendo.net/2007/01/15/wii-sports-experiment-results/

Deterding, S., Dixon, D., Nacke, L., O'Hara, K., \& Sicart, M. (2011). Gamification: Using Game Design Elements in Non-Gaming Contexts. In Proceedings of the SIGCHI conference on Human factors in computing systems, Vancouver, Canada.

Dourish, P., \& Bell, G. (2011). Divining a digital future: mess and mythology in ubiquitous computing: The MIT Press.

Duckworth, J. (2010). Embodiment and Play in designing an interactive art system for movement rehabilitation. Second Nature: International journal of creative media, 2(1), 120-137.

EA Sports. (2010). Grand Slam Tennis. Retrieved from http://tennis.easports.com/

ERCollab: Exergaming Research Collaboration. http://health.groups.yahoo.com/group/ERCollab. Retrieved from http://health.groups.yahoo.com/group/ERCollab

Etter, R., Specht, M. (2005). Melodious Walkabout - Implicit Navigation with Contextualized Personal Audio Contents. In Third International Conference on Pervasive Computing, Munich, Germany.

eyetoykinetic.com. EyeToy Kinetic. Retrieved from eyetoykinetic.com

Fox, J., \& Bailenson, J. N. (2009). Virtual self-modeling: The effects of vicarious reinforcement and identification on exercise behaviors. Media Psychology, 12(1), 1-25.

Gamersize. (n.d.). Gamersize. Retrieved from http://gamersize.net 
Graves, L., Stratton, G., Ridgers, N. D., \& Cable, N. T. (2007). Comparison of energy expenditure in adolescents when playing new generation and sedentary computer games: cross sectional study. BMJ, 335(7633), 12821284.

Hero, G. Guitar Hero. Retrieved from http://guitarhero.com

Hunicke, R. (2005). The case for dynamic difficulty adjustment in games. In Proceedings of the 2005 ACM SIGCHI International Conference on Advances in computer entertainment technology, Valencia, Spain. doi:10.1145/1178477.1178573

iFit. (2010). Retrieved from http://www.ifit.com/

Juul, J. (2009). A Casual Revolution: Reinventing Video Games and Their Players. Boston, MA, USA: MIT Press.

Kretchmar, R. (2005). Practical philosophy of sport and physical activity. Champaign, IL, USA: Human Kinetics Publishers.

Lanningham-Foster, L., Jensen, T. B., Foster, R. C., Redmond, A. B., Walker, B. A., Heinz, D., \& Levine, J. A. (2006). Energy Expenditure of Sedentary Screen Time Compared With Active Screen Time for Children. Pediatrics, 118(6), 1831-1835.

mapmyrun.com. (2010). MapMyRun. Retrieved from http://www.mapmyrun.com/

Marentakis, G. N., \& Brewster, S. A. (2006). Effects of feedback, mobility and index of difficulty on deictic spatial audio target acquisition in the horizontal plane. In Proceedings of the SIGCHI conference on Human Factors in computing systems, Montreal, Canada. doi:http://doi.acm.org/10.1145/1124772.1124826

Microsoft. (2010). Xbox Kinect. Retrieved from http://www.xbox.com/en-US/kinect

Mobile Adventure Walks. (2011). Retrieved from http://www.mobileadventurewalks.com/

Montola, M., Stenros, J., \& Waern, A. (2009). Pervasive Games: Theory and Design. Burlington, MA, USA: Morgan Kaufmann.

Mueller, F. (2009). Digital Sport: Merging Gaming with Sports to Enhance Physical Activities Such as Jogging. In Digital Sport for Performance Enhancement and Competitive Evolution: Intelligent Gaming Technologies (pp. 150-166). Retrieved from http://www.igi-global.com/reference/details.asp?id=33413

Mueller, F., Edge, D., Vetere, F., Gibbs, M. R., Agamanolis, S., Bongers, B., \& Sheridan, J. G. (2011). Designing Sports: A Framework for Exertion Games. In $\mathrm{CHI}$ '11: Proceedings of the SIGCHI Conference on Human Factors in Computing Systems, Vancouver, Canada.

Mueller, F., O'Brien, S., \& Thorogood, A. (2007). Jogging over a Distance: Supporting a "Jogging Together" Experience Although Being Apart. In $\mathrm{CHI}$ '07: Conference on Human Factors in Computing Systems, San Jose, CA, USA.

Mueller, F., Vetere, F., Gibbs, M. R., Agamanolis, S., \& Sheridan, J. (2010). Jogging over a Distance: The Influence of Design in Parallel Exertion Games. In ACM SIGGRAPH 2010, Los Angeles, USA.

Mueller, F., Vetere, F., Gibbs, M. R., Edge, D., Agamanolis, S., \& Sheridan, J. G. (2010). Jogging over a distance between Europe and Australia. In UIST '10. Proceedings of the 23nd annual ACM symposium on User interface software and technology, New York, New York, USA. doi:10.1145/1866029.1866062

Mueller, F., Vetere, F., Gibbs, M. R., Edge, D., Agamanolis, S., Sheridan, J. G., \& Heer, J. (2012). Balancing Exertion Experiences. In CHI '12: Proceedings of the SIGCHI Conference on Human Factors in Computing Systems, Austin, Texas.

Nintendo. Mario Party 8. Retrieved from http://www.nintendo.com/sites/mp8/

Nintendo. (n.d.). Wii. Retrieved from http://wii.nintendo.com 
Robertson, M. (2011). Can't play, won't play. Retrieved from http://www.hideandseek.net/2010/10/06/cant-play-wont-play/

RunKeeper. (2011). Retrieved from http://runkeeper.com

Salen, K., \& Zimmerman, E. (2003). Rules of Play: Game Design Fundamentals. Boston, MA, USA: The MIT Press.

Sheridan, J., \& Mueller, F. (2010). Fostering Kinesthetic Literacy Through Exertion Games. In Workshop on Whole-Body Interactions at CHI'10: International Conference on Human Factors in Computing Systems, Atlanta, USA.

Sinclair, J., Hingston, P., \& Masek, M. (2007). Considerations for the design of exergames. In Proceedings of the 5th international conference on Computer graphics and interactive techniques in Australia and Southeast Asia, Perth, Australia.

Tacx. (2009). Tacx Virtual Reality. Retrieved from http://www.tacxvr.com

Weinberg, R. S., \& Gould, D. (2006). Foundations of Sport and Exercise Psychology. Champaign, IL, USA: Human Kinetics.

Weiser, M. (1993). Some computer science issues in ubiquitous computing. Communications of the ACM, 36(7), 75-84.

Wikipedia contributors. (2011). Retrieved from http://en.wikipedia.org/wiki/Wii_Remote_Plus\#Wii_Remote_Plus

Zombies, Run! (2011). Retrieved from http://www.zombiesrungame.com/ 